fund, Memorial University of Newfoundland; the Gerontology Centre; Abbott Laboratories, Montreal, Canada; and Ross Laboratories, Columbus, Ohio.

1 Chandra RK. Nutrition, immunity and infection: present knowledge and future directions. Lancet 1983; ; :688-91.

2 Chandra RK, ed. Nutrition, immunity and illness in the elderly. New York: PerRivlin RS, Young $1985: 1-100$

vin RS, Young EA, eds. Symposium on evidence relating selected vitamins and minerals to health and disease in the

4 Chandra RK, Joshi $P$, Au B, Woodford G, Chandra $S$. Nutrition and imm competence of the elderly. Effect of short term nutritional supplementation on
cell-mediated immunity and lymphocyte subsets. Nutr Res 1982.2:223-32 5 Schneider EL. Infectious disease in the elderly. Ann Intern Med 1983;98:395-400.

(Accepted 12 June 1985)

Departments of Medicine, Paediatrics, and Biochemistry, Memorial University of Newfoundland, Canada

RANJIT KUMAR CHANDRA, MD, FRCP(C), professor

SHAKUNTLA PURI, MD, postdoctoral fellow

Correspondence and requests for reprints to: Professor $\mathrm{R} \mathrm{K}$ Chandra, Health Sciences Centre, St John's, Newfoundland A1B 3V6, Canada.

\section{Fast atrial fibrillation induced by treatment of psoriasis with azathioprine}

We describe a patient with psoriasis who developed fast atrial fibrillation in an idiosyncratic reaction to azathioprine.

\section{Case report}

A 60 year old man was referred for treatment of widespread psoriasis. He had suffered from the condition for over 20 years and had received various topical treatments and photochemotherapy. The response to psoralens and ultraviolet $A$ treatment had been unsatisfactory after some initial improvement, and immediately before his referral to our department he had been using potent topical steroid ointments. He had a history of heavy alcohol consumption. Examination showed widespread confluent plaques of psoriasis, which had a glazed, thinned appearance. No abnormal cardiovascular signs were found, and he was in sinus rhythm at 60 beats/min with a blood pressure of $140 / 90 \mathrm{~mm} \mathrm{Hg}$. An electrocardiogram on admission (figure $(A)$ ) and chest $x$ ray film were normal. We considered him to be unsuitable for routine treatment with dithranol, and azathioprine $50 \mathrm{mg}$ three times daily was started. He received no other drug.

Four days later he became febrile, and over the next 48 hours his temperature reached $40^{\circ} \mathrm{C}$ and was accompanied by a sinus tachycardia. Azathioprine was stopped, and repeated cultures of blood and urine yielded negative results. Three days later, after the fever and tachycardia had completely settled, azathioprine was reintroduced. Within five hours he developed rigors and a fever $\left(39^{\circ} \mathrm{C}\right)$ and was found to be in fast atrial fibrillation (figure $(B)$ ). Azathioprine was again immediately stopped, and the atrial fibrillation resolved within hours on bed rest. Tests of thyroid function yielded normal results, and serum electrolyte concentrations and a later electrocardiogram (figure $(C)$ ) were normal.

Subsequent inquiries about previous treatment at another hospital showed that he had developed a fever while taking azathioprine seven years before. It had recurred on reintroduction of the drug.

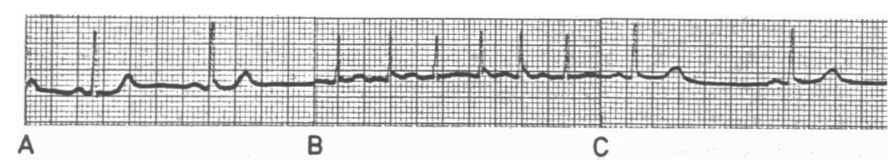

Electrocardiogram (lead II) $(A)$ on admission; $(B)$ five hours after azathioprine $50 \mathrm{mg}$; and $(C)$ after bed rest and withdrawal of azathioprine.

\section{Comment}

Azathioprine is a widely used immunosuppressive agent that is effective in severe and disabling psoriasis. ${ }^{1}$ Toxic effects include depression of bone marrow, hepatotoxicity, gastrointestinal upsets, reduced resistance to infection, malignant tumours, and teratogenicity. Because patients show better tolerance of and clinical response to methotrexate azathioprine has become a second line agent in severe psoriasis that is used when other treatments have failed or are contraindicated.
Febrile reactions to azathioprine have been reported and can, as in our case, develop within hours after administration of the drug. The mechanism is unclear, but the fever may be associated with polyarthritis. We have found no reports of cardiac side effects of azathioprine, and neither the manufacturers (Wellcome Medical Division, United Kingdom) nor the Committee on Safety of Medicines has received reports of any such side effects in toxic reactions. Episodes of atrial fibrillation in normal hearts can be precipitated by other drugs, including alcohol, ${ }^{2}$ inappropriate thyroxine treatment, ${ }^{3}$ and nicotine, ${ }^{4}$ as well as by non-cardiac events such as surgery, pneumonia, burns, mediastinal carcinoma, lymphoma, pulmonary embolism, and renal and biliary colic. ${ }^{5}$ There was no precipitating event in our patient other than the administration of azathioprine, and the heart was clinically and electrocardiographically normal before and after the episode.

It is important for physicians using azathioprine to be aware of this serious but reversible adverse effect.

$1 \mathrm{Du}$ Vivier A, Munro DD, Verbov J. Treatment of psoriasis with azathioprine. Br Med f $1974 ; \mathrm{i}: 49-51$.

Thornton JR. Atrial fibrillation in healthy non-alcoholic people after an alcoholic binge. Lancet $1984 ;$ ii:1013-4

fliman
E. Auricular fi

on Ahn B. A further riedberg CK. Diseases of the 1953;145:28-33.

Accepted 9 August 1985)

Department of Dermatology, Royal Free Hospital, London NW3 2QG H J DODD, MRCP, senior registrar F M TATNALL, MRCP, registrar I SARKANY, FRCP, consultant

Correspondence to: Dr Dodd.

\section{Orchidopexy: theory and practice}

Only $32 \%$ of orchidopexies are done by the recommended age of 5 years. The number of operations is four times greater than the incidence of undescended testis, suggesting that boys with retractile testes have unnecessary surgery. We decided to investigate further.

\section{Present survey and results}

With the help of colleagues in 12 hospitals in different parts of Britain we recorded the ages of 1285 boys undergoing orchidopexy between 1981 and 1983. The proportions who had surgery before the age of 5 ranged from $7 \%$ to $51 \%$, with a mean of $32 \%$.

We took the incidence of undescended testis as $1 \%$ and calculated the expected number of cases for six district hospitals with known catchment populations. In these the numbers of orchidopexies were two to four times greater than expected (table).

All general practitioners in the Medway district were asked when they would refer boys with undescended testis for surgery; of the 92 who replied, $69(75 \%)$ said that they would do so before the age of 5 years.

We examined the hospital and, wherever possible, neonatal and community child health records of 58 boys who had had orchidopexies in 1983. In 15 cases the diagnosis had been made at the neonatal examination but none of these children, if otherwise well, had been followed up by paediatricians. In 22 cases the referral letter stated that the diagnosis had been made by a community medical officer, but in many cases the separate neonatal, hospital, child health clinic, and school medical records made it impossible to elicit this information.

Among these cases we found two where the testes had been noted to be

Expected and actual numbers of orchidopexies performed in six district general hospitals

\begin{tabular}{cccc}
\hline & No of male & \multicolumn{2}{c}{ No of orchidopexies a year } \\
\cline { 3 - 4 } Hospital & births a year & Expected & Performed \\
\hline 1 (Medway) & 2500 & 25 & 52 \\
2 & 1000 & 10 & 22 \\
3 & 1900 & 19 & 43 \\
4 & 1800 & 18 & 65 \\
5 & 2000 & 20 & 62 \\
\hline 6 & 1400 & 14 & 14070 \\
\hline
\end{tabular}

